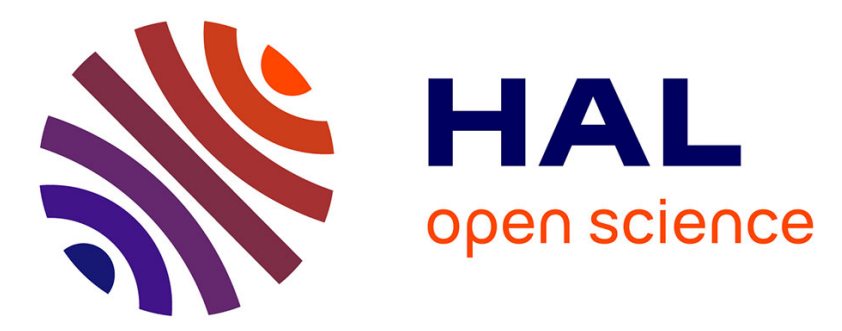

\title{
Primary appendiceal carcinoma - epidemiology, surgery and survival: results of a German multi-center study
}

F. Benedix, A. Reimer, I. Gastinger, P. Mroczkowski, H. Lippert, R. Kube

\section{To cite this version:}

F. Benedix, A. Reimer, I. Gastinger, P. Mroczkowski, H. Lippert, et al.. Primary appendiceal carcinoma - epidemiology, surgery and survival: results of a German multi-center study. EJSO - European Journal of Surgical Oncology, 2010, 36 (8), pp.763. 10.1016/j.ejso.2010.05.025 . hal-00608928

\section{HAL Id: hal-00608928 \\ https://hal.science/hal-00608928}

Submitted on 16 Jul 2011

HAL is a multi-disciplinary open access archive for the deposit and dissemination of scientific research documents, whether they are published or not. The documents may come from teaching and research institutions in France or abroad, or from public or private research centers.
L'archive ouverte pluridisciplinaire $\mathbf{H A L}$, est destinée au dépôt et à la diffusion de documents scientifiques de niveau recherche, publiés ou non, émanant des établissements d'enseignement et de recherche français ou étrangers, des laboratoires publics ou privés. 


\section{Accepted Manuscript}

Title: Primary appendiceal carcinoma - epidemiology, surgery and survival: results of a German multi-center study

Authors: F. Benedix, A. Reimer, I. Gastinger, P. Mroczkowski, H. Lippert, R. Kube

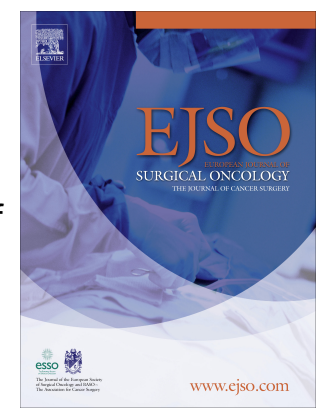

PII: S0748-7983(10)00154-X

DOI: $\quad$ 10.1016/j.ejso.2010.05.025

Reference: YEJSO 2987

To appear in: European Journal of Surgical Oncology

Received Date: 18 December 2009

Revised Date: 15 May 2010

Accepted Date: 27 May 2010

Please cite this article as: Benedix F, Reimer A, Gastinger I, Mroczkowski P, Lippert H, Kube R. Primary appendiceal carcinoma - epidemiology, surgery and survival: results of a German multi-center study, European Journal of Surgical Oncology (2010), doi: 10.1016/j.ejso.2010.05.025

This is a PDF file of an unedited manuscript that has been accepted for publication. As a service to our customers we are providing this early version of the manuscript. The manuscript will undergo copyediting, typesetting, and review of the resulting proof before it is published in its final form. Please note that during the production process errors may be discovered which could affect the content, and all legal disclaimers that apply to the journal pertain. 


\section{Primary appendiceal carcinoma -}

\section{epidemiology, surgery and survival:}

\section{results of a German multi-center study}

F. Benedix ${ }^{1,3 *}$, A. Reimer ${ }^{2,3^{*}}$, I. Gastinger ${ }^{2,3}$, P. Mroczkowski ${ }^{1,3}$, H. Lippert ${ }^{1,3}$, R. Kube ${ }^{2,3}$; the Study Group "Colon/Rectum Carcinoma (Primary Tumor)“

1) Department of General, Visceral and Vascular Surgery, Otto-von-Guericke University Magdeburg, Leipziger Str. 44, 39120 Magdeburg, Germany

2) Department of Surgery, Carl-Thiem Hospital Cottbus, Thiemstraße 111, Cottbus, 03048, Germany

3) Institute for Quality Assurance in Operative Medicine, Otto-von-Guericke University Magdeburg, Leipziger Str. 44, Magdeburg, 39120, Germany

* Benedix F \& Reimer A contributed equally to this work.

\section{Corresponding author:}

Frank Benedix, M.D.

Department of General, Visceral and Vascular Surgery

Otto-von-Guericke University Magdeburg

Leipziger Str. 44

D-39120 Magdeburg, Germany

Tel. +49-391-6715500

Fax: +49-391-6715570

E-mail: frankbenedix@gmx.de 


\section{Abstract}

Background. While carcinoma of the colon is a common malignancy, primary carcinoma of the appendix is rare. Many retrospective reviews outlined experience from different centers on appendiceal neoplasms. However, the study population is often small because it is so rare. The aim of this study was to analyze the type of surgery and survival of patients with appendiceal malignancies using data from a German multi-center observational study (31 341 patients).

Methods. During a five-year period, 196 consecutive patients with malignant appendiceal tumors were distributed into four groups: appendiceal carcinoids, adenocarcinoma, mucinous adenocarcinoma and adenosquamous carcinoma. The following parameters were analyzed: demographics, clinical presentation, comorbidities, type and appropriateness of surgery, final pathology and survival.

Results. Adenocarcinoma had the highest incidence (50.5\%). The most common presentation was that of acute appendicits. Mean age at presentation was youngest for carcinoid tumors. Carcinoid tumors had lowest tumor size and localized disease was present in $72.9 \%$. Metastatic spread at presentation was highest for adenosquamous and mucinous adenocarcinoma and each had a distinct pattern. Right hemicolectomy was performed in $71.4 \%$, limited resection in $11.7 \%$. Overall 5 year survival was $83.1 \%$ for carcinoid vs. $49.2 \%$ for non-carcinoid tumors. Histological subtype and tumor stage significantly affected survival.

Conclusions. Long-term outcome of carcinoid tumors is superior to noncarcinoid neoplasms. Among all appendiceal neoplasms, adenosquamous carcinoma is the rarest histological subtype which is most commonly associated with advanced tumor stage and worst prognosis. Appropriate oncologic resection is being performed in a significant percentage of cases in Germany. However, the high rate of right hemicolectomy in patients with small carcinoid tumors needs to be critically discussed.

Key words appendix - carcinoma - carcinoid - surgery - survival 


\section{Introduction}

Primary appendiceal tumors are rare entities, occuring in $<2 \%$ of all appendectomies with an age-adjusted incidence of 0.12 cases per 1,000,000 people per year [1-5]. The vast majority of these tumors are not diagnosed preoperatively - rather, they present with acute appendicitis or are detected as an incidental finding during exploration for another surgical pathology. Endocrine appendiceal tumors make up to $35-85 \%$ of all appendiceal neoplasms [6,7]. Most of these tumors are located at the tip of the appendix with a maximal diameter of $<1 \mathrm{~cm}$ in $60-80 \%$ of cases $[6,8]$. Metastatic disease in patients with tumors $<2 \mathrm{~cm}$ is rare. Five-year survival is $83 \%$ for all stages [9]. Primary adenocarcinoma of the appendix is a rare malignancy that constitutes less than $0.5 \%$ of all neoplasms of gastrointestinal origin [10]. These tumors arise in pre-existing adenomas, either by a cystic and colonic growth pattern. Cystic type is a mucin-producing tumor and is comparable to cystadenocarcinoma of the ovary, tending to rupture and spread throughout the abdominal cavity, resulting in pseudomyxoma peritonei. Colonic type is a polypoid or ulcerative tumor that develops from a tubular or tubulovillous adenoma $[6,11]$. It is comparable to adenocarcinoma of the colon. Mucinous histology has a better survival than colonic histology for appendiceal adenocarcinomas [9, 12]. Right hemicolectomy has been recommended for all adenocarcinomas. Surveillance for synchronous or metachronous tumors is warranted because the risk of a second primary malignancy in the gastrointestinal tract is significantly increased [13].

Adenosquamous carcinoma of the colon is defined as a neoplasm comprising adenocarcinoma and squamous cell carcinoma. The epithelium near the dentate line is anatomically capable of differentiating into both glandular and squamous epithelium. Primary adenosquamous carcinoma of the colon is rare at sites other than the lower rectum and anal canal and has a poor prognosis [14]. Adenosquamous carcinoma originating from the vermiform appendix has not been reported so far.

A number of retrospective reviews have outlined experience from different centers on appendiceal neoplasms. However, due to its rarity the number of patients in most of the reports is small. The purpose of this study was to examine all patients with neoplasms of the appendix in a German multi-center observational study. We evaluated epidemiology, patient characteristics, type of surgery, final pathology and survival for four distinct histological types of appendiceal tumors. Finally, we 
determined appropriateness of resection for appendiceal tumors according to current treatment guidelines in Germany. 


\section{Material and Methods}

Within the German multi-center observational study "Colon/Rectal Carcinoma (Primary Tumor)", data for 31341 patients with colon malignancy was acquired prospectively for a five year period.

A standardized questionnaire was completed at the hospital where the patient was treated, to record information about the patient, tumor, operation, final pathology and perioperative course. A follow-up questionnaire was completed by the patient's GP or, by the primary hospital itself to gather information on the last patient contact, life status, recurrence and if the patient died, the cause of death (tumor-related, tumorindependent). The study was conducted according to the Declaration of Helsinki for Biomedical Research. Participation was voluntary, evaluation was based on anonymous data, and the study involved observation only - it had no influence upon the choice or course of therapy. For these reasons, an Ethics Committee vote was not necessary. All patients gave written consent for data collection and anonymous evaluation.

\section{Inclusion criteria for the present analysis were:}

- Patients with malignant appendiceal tumors

- All patients recruited between January 1, 2000 and December 31, 2004

- Patients with surgical tumor-resection to ensure final pathology.

\section{Exclusion criteria were as follows:}

- Benign and non-epithelial tumors of the vermiform appendix

- Palliative surgery without resection of the tumor (i.e. bypass procedure).

Patients were distributed into four groups according to the histological subtype:

1) Appendiceal Carcinoids (AC)

2) Appendiceal Adenocarcinoma (aA)

3) Mucinous Appendiceal Adenocarcinoma (mAA)

4) Appendiceal Adenosquamous carcinoma (AAS)

The following parameters were analyzed for all patients: age, sex, body mass index (BMI), reason for admission, comorbidities, presence of synchronous distant metastases, type of surgery, necessity of multivisceral resection, final pathology, 5year-overall and disease-free survival (DFS). Histopathologically, all non-carcinoid 
neoplasms were classified according to the TNM and UICC-classification. All carcinoid tumors were categorized by maximum tumor size as $<1 \mathrm{~cm}, 1$ to $2 \mathrm{~cm}$ and $>$ $2 \mathrm{~cm}$. Furthermore, they were subdivided into three groups for staging purposes: 1) localized (the neoplasm confined entirely to the appendix); 2) regional (extension beyond the limits of the appendix directly into surrounding tissue and/or regional lymph nodes; 3) distant (tumors with metastatic spread). Overall and disease-free survivals were compared.

\section{Statistics}

All data was entered into an MS ACCESS data base and was processed by using the statistical package SPSS (version 13.0; SPSS Ltd., Chicago, IL, USA). Data is expressed as arithmetic means \pm standard deviation. The statistical significance of differences between the subgroups was estimated by one-factor analysis of variance (ANOVA) with subsequent multiple comparison according to Tukey or by Pearson's $\mathrm{X}^{2}$ test. Kaplan-Meier curves for survival were compared by the log-rank test. Differences between the groups were to be regarded as significant if $p<0.05$ was found in a two-sided test. 


\section{Results}

Between January 1, 2000 and December 31, 2004, all consecutive patients with colon malignancy $(n=31341)$ were analyzed for this study. Of those, $196(0.63 \%)$ had histologically confirmed malignant appendiceal tumors. Among all appendiceal neoplasms, the highest incidence in this study was demonstrated for aA $(n=99$; $50.5 \%)$, followed by AC $(n=48 ; 24.5 \%), \operatorname{mAA}(n=45 ; 23 \%)$ and AAS $(n=4 ; 2 \%)$. Study population / Demographics

Mean age at presentation for $A C$ (mean $51.1 \pm 17.4$ years) was significantly lower than for the other subtypes $(p<0.01)$. mAA had the highest proportion of female patients (51.1\%). A male predominance was noted for all other groups. Mean BMI did not differ significantly among all groups. $(p=0.396)$ (Table 1$)$

Clinical presentation / characteristics

Overall, 57 patients $(29.1 \%)$ were referred to the surgical department for secondary procedure after an appendiceal neoplasm was confirmed histologically following primary appendicectomy. Seventy-three patients (37.2\%) presented with signs and symptoms of an acute abdomen suggestive of acute appendicits. In 45 patients (23\%), the appendiceal lesion was discovered incidentially during another surgical procedure. The remaining 21 patients $(10.7 \%)$ were initially referred to the hospital with nonspecific abdominal pain or tumor-related symptoms for further investigation (Table 1). Comorbidities were significantly more common in patients with mAA (77.8\%), predominantly cardiovascular (53.3\%) and diabetes mellitus $(15.5 \%)(p<$ $0.01)$.

\section{Operative procedure}

Right hemicolectomy was performed in $71.4 \%$ of the cases (140 patients; AC: 35 ; aA: 67; mAA: 35 ; AAS: 3 ). This type of surgery was performed either due to apparent or suspected tumor intraoperatively or as intendedprocedure for patients admitted to the hospital with confirmed malignancy or as complementary procedure during the same hospital stay after primary appendicectomy. Twenty-three patients (11.7\%) underwent primary ileocecal resection, all for apparent or suspected tumor during initial surgery (AC: $3 ; \mathrm{aA}: 18 ; \mathrm{mAA}: 2$ ). Limited resection (cecal resection or appendicectomy) was performed in 11.7\% (23 patients, AC: 8; aA: 8; mAA: 6; AAS: 1). An extended right hemicolectomy became necessary in 9 patients (4.6\%, AC: 2 ; aA: $5 ; m A A: 2)$, a subtotal colectomy in one patient $(0.5 \%$, aA: 1$)$ due to synchronous colonic pathology. For the same reason, 5 patients had simultaneous sigmoid colon 
resection ( $A C: 1$; $a A: 3 ; m A A: 1), 3$ patients anterior resection $(a A: 1 ; m A A: 2)$ and one patient transverse colon resection (mAA: 1).

Multivisceral resection due to locally advanced tumor growth was performed in $10.4 \%$ of AC cases ( 2 small bowel, 2 ovary, 1 abdominal wall) and $16.2 \%$ of aA ( 4 small bowel, 3 ovary, 8 abdominal wall, 2 omentum, 1 uterus, partial resection of urinary bladder / liver / stomach: 1/2/1). Extended resection was most frequently $(33.3 \% ; p=$ 0.012 ) required in mAA patients ( 15 patients; 2 small bowel, 3 ovary, 1 abdominal wall, 3 omentum, 4 uterus, partial resection of urinary bladder 2). Complementary carcinologic resection was planned in a further 8 (4.1\%) patients (5 with primary appendicectomy, 3 with right hemicolectomy but inadequate lymph node dissection). Based on final pathology, adjuvant treatment with chemotherapy was initiated in $12.2 \%$ (aA: 15, mAA: 9 patients).

\section{Histopathologic staging / pathological analysis}

The mean tumor size at presentation was significantly lower for $\mathrm{AC}(1.8 \mathrm{~cm})$ compared to all other tumor subtypes $(p<0.01)$. (Table 2$)$. In $41.6 \%$ of all AC patients, tumor size was $<1 \mathrm{~cm}$. There were 14 patients $(29.1 \%)$ in each of the remaining groups: tumor diameter of $1-2 \mathrm{~cm}$ and $>2 \mathrm{~cm}$, respectively. Lymph node positive disease was diagnosed in $16.6 \%$. Stage distribution of localized appendiceal carcinoids, regionalized lesions and distant metastatic disease was $72.9 \%, 22.9 \%$ and $4.2 \%$, respectively. Lymphatic invasion was present in $10 \mathrm{AC}$ patients and was associated with advanced tumors. In all AC patients with distant metastastic disease and $62.5 \%$ of patients with lymph node positive disease, lymphatic invasion was found. Eight of the ten patients had a tumor size $>2 \mathrm{~cm}$. None of the patients with a tumor size $<1 \mathrm{~cm}$ had lymph node positive disease or lymphatic invasion. For noncarcinoid tumors, the highest proportion of locally advanced tumors was found for AAS (100\%), followed by mAA (88.9\%). Lymph node positive disease was most frequently diagnosed in AAS (50\%), followed by aA (36.4\%). The lowest rate of lymphatic tumor spread was found for $\mathrm{AC}(16.6 \%)(p=0.052)$. Analysis of tumor differentiation revealed the highest proportion of poorly differentiated carcinomas for AAS (75\%), whereas mAA had the highest number of well differentiated tumors $(24.4 \% ; p=0.042)$ (Table 2). Incidence of synchronous metastases at presentation was highest for AAS (50\%), followed by mAA (28.9\%) and aA (23.2\%). In AC, presence of distant metastatic disease was found in $4.2 \%$ and only for tumors with a 
diameter $>2 \mathrm{~cm}$. Distinct results were also found regarding the pattern of metastatic spread. Among all patients with metastatic disease, hepatic metastases were more commonly associated with $\mathrm{AC}(50 \%)$ and $\mathrm{aA}(43.5 \%)$ compared with mAA $(7.7 \%)$ and AAS $(0 \%)(p=0.049)$. By contrast, peritoneal carcinomatosis was found in both cases of metastatic AAS and $76.9 \%$ of the metastatic mAA cases (Table 3). Rate of synchronous colon neoplasms was $6.2 \%, 10.1 \%$ and $8.9 \%$ for $\mathrm{AC}$, aA and $\mathrm{mAA}$, respectively.

\section{Appropriateness of surgical resection/therapy}

Appendicectomy or cecal resection alone as definitive surgical procedure was performed on $23(11.7 \%)$ patients (AC: 8, aA: 8, mAA: 6; AAS: 1). Seven patients (aA: 4, mAA: 2, AAS: 1) had palliative appendicectomy/cecal resection due to distant metastastic disease. Six patients had $A C$ with a tumor diameter of $<1 \mathrm{~cm}$. In five (2 $\mathrm{AC}$ with a tumor size $>2 \mathrm{~cm}, 1 \mathrm{~T} 2 \mathrm{aA}, 1 \mathrm{~T} 2 \mathrm{mAA}$, and $1 \mathrm{~T} 4 \mathrm{mAA}$ ) complementary carcinologic resection was planned. The remaining 5 patients with appendiceal tumors (AC > 2cm; T1\&T2 aA; T2\&T3 mAA) and local resection alone had no oncological surgery.

Ileocecal resection was performed in 23 (11.7\%) patients. Of those, 8 patients had distant metastatic disease and two $A C$ with a tumor diameter $<2 \mathrm{~cm}$. The remaining 13 patients had $\mathrm{AC}>2 \mathrm{~cm}$ ( 1 patient) and aA (12 patients, T1 x 1, T2 x 4, T3 x 6; T4 x $1 ; \mathrm{N} 1 \times 2)$.

According to current guidelines, right hemicolectomy should be performed for all noncarcinoid invasive tumors and $A C$ tumors $>2 \mathrm{~cm}$. Eighteen patients $(9.2 \%)$ in this study fulfilled these requirements, but did not undergo oncologic surgery. For small ( $\leq$ $1 \mathrm{~cm}$ ) carcinoid tumors, appendicectomy is recommended, however, of the 20 patients with an AC tumor $<1 \mathrm{~cm}$ in this study, $60 \%$ underwent unnecessary right hemicolectomy. None of these patients was diagnosed with concomitant lymphatic invasion.

According to current guidelines, all patients with UICC stage III colon adenocarcinoma should receive adjuvant chemotherapy. In this study, only 24 out of $29(82.7 \%)$ patients with lymph node positive disease had chemotherapy following curative surgical resection. 


\section{Survival}

Complete and plausibility-checked follow-up data was available for $89.3 \%$ of all 196 patients. The median follow-up time was 40.6 months. Perioperative mortality rate was $2.5 \%$ (5 patients). Overall 5 -year survival was $83.1 \%$ for carcinoid vs. $49.2 \%$ for non-carcinoid tumors (aA: $47.5 \%$, mAA: $50.9 \%)(p=0.01)$ (Fig.1). Two of four patients with AAS died within 25.9 months of diagnosis. Tumor stage (localized, regionalized and distant metastatic disease) and tumor size significantly affected survival in AC with overall 5-year survival rates of $92.2 \%, 80 \%$ and $50 \%$ (tumor stage), and $100 \%$, $80 \%$ and $50 \%$ (tumor size), respectively $(p<0.01)$. Patients with mAA fared better than those with aA when analyzed with respect to UICC stage except for stage III (100\% (I), $79.3 \%$ (II), $25.4 \%$ (III), 17.9\% (IV) vs. $87.5 \%, 58.7 \%, 38.8 \%, 12.5 \%$ ). Similarly, histological subtype significantly affected DFS with 5-year survival rates of $87.7 \%$ for carcinoid vs. $53.5 \%$ for noncarcinoid tumors ( $p<0.01$ ) (Fig. 2 ). Of the four patients with AAS, two were diagnosed with primary metastatic disease. In the remaining two, there is no evidence of tumor recurrence 19 and 29 months, respectively, after curative resection. To determine the influence of type of surgery on DFS, all patients with limited resection were compared with those who underwent appropriate oncologic resection with curative intent. Surprisingly, of the latter group, $42 \%$ developed recurrent disease whereas only $22.2 \%$ of the patients with limited resection had a local recurrence or distant metastatic spread. However, these results may not be representative because median follow-up for patients with limited resection was shorter (31.0 months) and sample size was significantly smaller. 


\section{Discussion}

While carcinoma of the colon is a common malignancy, primary carcinoma of the appendix is rare and is a somewhat different entity, particularly in presentation and histological type. It constitutes $<0.5 \%$ of all gastrointestinal neoplasms [13]. The lifetime incidence of malignant appendiceal tumors ranges from 0.2 to $0.5 \%$ [15]. The current study evaluates the epidemiology, type of surgery, final pathology and survival outcome for four distinct histological types of appendiceal carcinoma using data from a multi-centered observational study. Among all colon carcinomas, the incidence of malignant appendiceal tumors in this report was $0.63 \%$. Incidence of noncarcinoid appendiceal neoplasms was higher than that of carcinoid tumors, which is in line with the results of the two largest series of appendiceal tumors [4,9]. Mean age at presentation for noncarcinoid appendiceal neoplasms ranged from 59 to 63 years in most reports - consistent with the findings in this study $[4,7,9]$. Although patients with malignant carcinoids were significantly younger at the time of diagnosis, mean age was higher than reported by others $[4,7,9]$. This can be attributed to the fact that goblet cell carcinoids, which present at a higher age, were not separately analyzed in this study. Gender distribution for noncarcinoid tumors was comparable with other reports $[4,9]$. By contrast, there was a male predominance for carcinoid tumors, which has also been observed in smaller series [16-18]. This is possibly related to analysis of both, classical carcinoids and adenocarcinoids as one group and to the relatively low number of patients. Clinical presentation varied in this study. Most commonly, patients presented with symptoms and signs suggesting acute appendicitis, nonspecific pain or tumor-related symptoms, similar to results of other studies $[2,6,12,13]$. However, the number of patients with a preoperative diagnosis of acute appendicitis was lower compared with other reports, which may be attributed to the fact that nearly one third of the patients were referred for complementary surgery. In these patients, initial presenting symptoms were not recorded. The higher rate of concomitant medical disease for patients with noncarcinoid neoplasms in this study is most likely related to the higher age at the time of diagnosis.

For appendiceal carcinoids, the stage distribution was $72.9 \%$ for localized, $22.9 \%$ for regionalized lesions and $4.2 \%$ for distant metastatic disease, which is comparable with those reported by others $[7,9]$. Lymphatic invasion was found to be associated with advanced tumor growth and metastatic disease and thus, may be of predictive value. Therefore, based on these results, in every patient with lymphatic invasion on 
final pathology, complementary surgery should be strongly considered. For noncarcinoid tumors, mAA had a higher proportion of locally advanced tumors, whereas aA was more frequently diagnosed with lymph node positive disease. This is in agreement with the results of other studies $[4,9,13]$. Analysis of tumor grade revealed the significantly highest rate of well differentiated carcinoma for mAA, which is not being reflected in proportion to locally advanced and metastatic tumors. The results provide further evidence that there are specific differences in the molecular biological pattern between $\mathrm{mAA}$ and aA which subsequently cause a different carcinogenesis and development of biologically different cancers. Appendiceal carcinoid tumors exhibit little metastatic potential and therefore, rarely present with metastases $[19,20]$. They usually metastasize to the regional lymph nodes rather than to the liver. The tumor size is of predicting value for metastatic disease. The calculated risk of metastases from tumors $<1 \mathrm{~cm}$ is zero, while a definite increase occurs with a tumor size $>2 \mathrm{~cm}$, with a rate of metastases of $20 \%$ $[16,21]$. In this report, only tumors $>2 \mathrm{~cm}$ had distant metastatic disease at the time of diagnosis with a rate of $14.3 \%$. By contrast, noncarcinoid neoplasms of the appendix exhibit a more aggressive behaviour and are more likely to develop metastatic disease. $\mathrm{mAA}$ were reported to have a higher rate of metastases than $\mathrm{aA}$, which is in agreement with our results $[4,13]$. In $\mathrm{mAA}$, perforation will often result in pseudomyxoma peritonei, that is, peritoneal tumor implantation with free intraperitoneal mucous containing cellular elements. Apart from AAS, mAA had the highest rate of peritoneal carcinomatosis in this study. Peritoneal cavity was also the most common location for metastases of mAA in a study by Nitecki et al. [13]. In contrast to our results, no lymphatic or blood-borne metastases were found in that report. It has been recognized that patients with pseudomyxoma peritonei and mucinous tumors of the ovaries often have mucinous tumors of the appendix and that the ovarian tumors are probably secondary to the appendiceal tumor [22]. This is in agreement with our results, with the highest rate of metastases to the ovaries for mAA. In a study by Cortina et al., ovaries were the second most common location of metastases in patients with mAA [12]. In contrast, patients with appendiceal adenocarcinoma were more frequently found to have liver metastases in this study, suggesting a different pattern of metastatic spread, and thus supporting the notion that $\mathrm{mAA}$ and $\mathrm{aA}$ are two distinct morphologies with different biological behavior. 
According to the current recommendations, all noncarcinoid tumors should undergo right hemicolectomy, whereas the type of surgical intervention for carcinoid tumors of the appendix depends on size, high grade or high mitotic index, mesoappendiceal invasion, location, and presence of goblet cell histology $[9,23,24]$. Local resection is adequate for small carcinoid tumors $(<2 \mathrm{~cm})$ at the appendiceal tip, with no evidence of local spread [23]. However, adequate therapy of patients with tumor size from 1 to $2 \mathrm{~cm}$ in diameter remains a matter of controversy since there is a risk of regional metastases. On the other hand, right hemicolectomy is a significant abdominal procedure with an associated risk, especially in the elderly or infirm [23]. Comparing the risk of right hemicolectomy with the survival figures for patients with lesions smaller than $2 \mathrm{~cm}$, a more conservative approach seems appropriate in most circumstances. In those with tumors of $1-2 \mathrm{~cm}$ towards the tip of the appendix, with typical carcinoid histology, no mesoappendiceal invasion and low proliferative index, a right hemicolectomy does not appear to be justified [23]. Roggo et al. concluded that it would be reasonable to offer right hemicolectomy only to younger patients [19]. Goede et al. described acceptable indications for re-intervention: lesion larger than $2 \mathrm{~cm}$ in diameter, histological evidence of of mesoappendiceal extension, tumors at the base of the appendix with positive margins or involvement of the cecum, highgrade malignant carcinoid and goblet-cell adenocarcinoids [23]. In addition, angiolymphatic invasion was demonstrated in this study to be associated with advanced tumor growth and metastatic disease. Therefore, we would also recommend complementary surgery in these patients providing they are in a reasonable medical condition. Timing of secondary hemicolectomy should be within 3 months after appendicectomy and can safely be performed by laparoscopic approach. There is no data to support that a two-step approach may negatively affect the prognosis [24].

Adenocarcinoma of the appendix should be treated with right hemicolectomy due to the high rate of invasion and nodal metastases [13]. Patients with primary appendicectomy are best managed by reoperation and a formal right hemicolectomy because of the risk of overlooked nodal metastases which have been reported to be approximately $38 \%$ [13]. Adjuvant therapy is similar to what is recommended for colon adenocarcinomas. Thus, all patients with lymphatic involvement should receive adjuvant chemotherapy [6]. The treatment of appendiceal mucinous-type tumors appears to be more controversial. However, a few studies reported a survival 
advantage between right hemicolectomy and simple appendicectomy $[12,13]$. Moreover, the results of this study support a more aggressive approach even if metastatic disease is present due to a better survival compared to patients with adenocarcinoma. Although there is a tumor spread throughout the peritoneal cavity with superficial invasion in up to $65 \%$ in patients with pseudomyxoma peritonei, deep visceral invasion is rare $[25,26]$. Therefore, oncologic right hemicolectomy, tumor debulking and removal of all mucinous ascites seem to be justified in these patients. We agree that routine oophorectomy, especially if post-menopausal, should at least be considered in all cases with adenocarcinoma, particularly in mAA due to a high rate of ovarian metastases.

The results of this study suggest that appropriate oncologic resection and adjuvant chemotherapy are being performed for patients with appendiceal carcinoma in a significant percentage of cases in Germany. However, undertreatment of patients was still apparent in some cases as well as overtreatment in a high proportion of patients with carcinoid tumors $<1 \mathrm{~cm}$ who underwent unnecessary right hemicolectomy, significantly increasing perioperative morbidity. It is worth mentioning that patients with appendiceal carcinoma have a notable risk of developing a synchronous colorectal neoplasm which was highest in this study for patients with mucinous adenocarcinoma. Therefore, careful diagnostic work-up is required before complementary surgery is performed.

Most patients with $\mathrm{AC}$ have a favorable prognosis with a 5-year survival of $83 \%$ for all stages [9]. Patients with local disease are reported with a 5-year survival rate ranging from 92 to $94 \%$, those with regional metastases 81 to $84 \%$, and the few with distant metastases 31 to $33 \%[7,9,27]$. This was confirmed by our study, but with a better outcome for patients with metastatic disease, possibly related to the relatively small number of cases. Tumor size was also found to be significantly related to survival with a 5 -year survival rate of $100 \%$ for tumors $<1 \mathrm{~cm}$. Accordingly, histological subtype also affected DFS with the highest survival rate for patients with AC. The excellent survival of appendiceal carcinoids may reflect the specific histopathogenesis (neuroectodermal origin) [7]. Carcinoids of the appendix mostly develop from the subepithelial-endocrine cells and are immunoreactive for S-100, a protein normally present in Schwann cells, indicating that anatomically they belong rather to the peripheral nervous system and the ectoderm [19]. The outcome of 
patients with noncarcinoid tumors is much worse with a 5-year survival rate ranging from 44 to $55 \%$ for all stages in most of the reports including the current study $[9,13]$. Among all noncarcinoid tumors, mAA was demonstrated to have highest survival rate. The difference was most pronounced in patients with metastatic disease in both our series and other reports [4,9]. This underlines the importance and potential benefit of an aggressive approach to the management of $\mathrm{mAA}$ - even if peritoneal carcinomatosis is present - with right hemicolectomy, and debulking and removal of all mucinous ascites where appropriate.

Adenosquamous carcinoma of the colon is extremely rare and represents $0.025 \%$ to $0.1 \%$ of all colorectal malignancies [28]. They occur most frequently in the lower rectal area. To the best of our knowledge, there are no reports in the English literature about adenosquamous carcinoma arising from the vermiform appendix. Among all colon malignancies and appendiceal tumors, incidence of this rare histological subtype in this study was $0.01 \%$ and $2.04 \%$, respectively. Mean age at presentation for colorectal AAS ranges from 62.5 to 63.3 years, which is higher than observed for appendiceal AAS in this report $[14,29,30]$. We observed a male predominance, which was also demonstrated for colorectal AAS [14,30]. Presenting symptoms and signs of colorectal AAS are similar to those for patients with adenocarcinoma of the colon, whereas half of the patients with appendiceal AAS - as most of the patients with appendiceal neoplasm - presented with symptoms suggestive of acute appendicitis. All patients with AAS in this study had locally advanced tumors at the time of diagnosis with $50 \%$ having lymph node positive disease and $50 \%$ distant metastatic spread to the peritoneum. There was also a high rate of poorly differentiated carcinoma $(75 \%)$. Comparable rates of lymph node and distant metastases have been reported for colorectal AAS [14,30]. Accordingly, prognosis is much worse than that of adenocarcinoma alone with $52 \%$ not surviving beyond one year [30]. In this study, $50 \%$ of all patients died within 26 month after initial presentation.

There are some limitations to the study that should be acknowledged. The data used for this study was collected from many hospitals in Germany and include diagnoses of numerous pathologists. Thus, there are no effective means of establishing the reliability of the tissue diagnoses. Secondly, no data regarding location of 
appendiceal carcinoid, presence of mesoappendiceal invasion and proliferative index were recorded. Furthermore, carcinoid tumors were analyzed as one group without distinguishing between classical carcinoid and goblet-cell carcinoid which may have influenced results, especially in terms of mean age and survival.

Primary carcinoma of the appendix remains a rare entity, which is difficult to diagnose preoperatively and classically presents as inflammatory disease. Long-term outcome of carcinoid tumors is superior to noncarcinoid neoplasms, and is significantly affected by tumor stage and tumor size. Lymphatic invasion should be considered as an additional risk factor. Among all appendiceal neoplasms, adenosquamous carcinoma is the rarest histological subtype, which is most commonly associated with advanced tumor stage at the time of diagnosis and thus, with worst prognosis. The results of the study suggest that appropriate oncologic resection is being performed in a significant percentage of cases in Germany. However, the high rate of unnecessary right hemicolectomy in patients with small carcinoid tumors as well as the undertreatment of appendiceal cancer patients needs to be critically assessed and should encourage surgeons to improve adherence to current treatment guidelines.

\section{CONFLICT OF INTEREST STATEMENT}

The authors declare that they have no conflicts of interest. 


\section{References}

[1] Connor SJ, Hanna GB, Frizelle FA. Appendiceal tumors: retrospective clinicopathologic analysis of appendiceal tumors from 7,970 appendectomies. Dis Colon Rectum 1998; 41 : 75-80.

[2] Bucher P, Mathe Z, Demirag A, Morel P. Appendix tumors in the era of laparoscopic appendectomy. Surg Endosc 2004; 18: 1063-6.

[3] Iwuagwu OC, Jameel JK, Drew PJ, Hartley JE, Monson JR. Primary carcinoma of the appendix - Hull series. Dig Surg 2005; 22: 163-7.

[4] McCusker ME, Coté TR, Clegg LX, Sobin LH. Primary malignant neoplasms of the appendix: a population-based study from the surveillance, epidemiology and end-results program, 1973-1998. Cancer 2002; 94: 3307-12.

[5] von Suchodoletz H, Völzer H. Distinctive features of appendicitis in childhood. Zentralbl Chir 2008; 133: 554-8.

[6] O'Donnell ME, Badger SA, Beattie GC, Carson J, Garstin WI. Malignant neoplasms of the appendix. Int J Colorectal Dis 2007; 22: 1239-48.

[7] Sandor A, Modlin IM. A retrospective analysis of 1570 appendiceal carcinoids. Am J Gastroenterol 1998; 93: 422-8.

[8] Stinner B, Rothmund M. Neuroendocrine tumours (carcinoids) of the appendix. Best Pract Res Clin Gastroenterol 2005; 19: 729-38.

[9] McGory ML, Maggard MA, Kang H, O'Connell JB, Ko CY. Malignancies of the appendix: beyond case series reports. Dis Colon Rectum 2005; 48: 2264-71.

[10] Chang P, Attiyeh FF. Adenocarcinoma of the appendix. Dis Colon Rectum 1981; 24: 176-80. 
[11] Deans GT, Spence RA. Neoplastic lesions of the appendix. Br J Surg 1995; 82: 299306.

[12] Cortina R, McCormick J, Kolm P, Perry RR. Management and prognosis of adenocarcinoma of the appendix. Dis Colon Rectum 1995; 38: 848-52.

[13] Nitecki SS, Wolff BG, Schlinkert R, Sarr MG. The natural history of surgically treated primary adenocarcinoma of the appendix. Ann Surg 1994; 219: 51-7.

[14] Yokoi K, Tanaka N, Furukawa K, Seya T, Ohaki Y, Tajiri T. Case of adenosquamous carcinoma of the ascending colon. J Nippon Med Sch 2008; 75: 242-6.

[15] Cerame MA. A 25-year review of adenocarcinoma of the appendix. A frequently perforating carcinoma. Dis Colon Rectum 1988; 31: 145-50.

[16] Tchana-Sato V, Detry O, Polus M et al. Carcinoid tumor of the appendix: a consecutive series from 1237 appendectomies. World J Gastroenterol 2006; 12: 6699-701.

[17] In't Hof KH, van der Wal HC, Kazemier G, Lange JF. Carcinoid tumour of the appendix: an analysis of 1,485 consecutive emergency appendectomies. J Gastrointest Surg 2008; 12 : 1436-8.

[18] Graham RP, Williams NP, West KA. Primary epithelial tumours of the appendix in a black population: a review of cases. World J Gastroenterol 2009; 15: 1472-4.

[19] Roggo A, Wood WC, Ottinger LW. Carcinoid tumors of the appendix. Ann Surg 1993; 217: $385-90$.

[20] Lehner F, Ramackers W, Bektas H, Becker T, Klempnauer J. Liver resection for noncolorectal, non-neuroendocrine liver metastases--is hepatic resection justified as part of the oncosurgical treatment? Zentralbl Chir 2009; 134: 430-6.

[21] Thompson GB, van Heerden JA, Martin JK Jr, Schutt AJ, Ilstrup DM, Carney JA. Carcinoid tumors of the gastrointestinal tract: presentation, management, and prognosis. Surgery 1985; 98: 1054-63. 
[22] Young RH, Gilks CB, Scully RE. Mucinous tumors of the appendix associated with mucinous tumors of the ovary and pseudomyxoma peritonei. A clinicopathological analysis of 22 cases supporting an origin in the appendix. Am J Surg Pathol 1991; 15: 415-29.

[23] Goede AC, Caplin ME, Winslet MC. Carcinoid tumour of the appendix. Br J Surg 2003; 90: 1317-22.

[24] Plöckinger U, Couvelard A, Falconi M et al. Consensus guidelines for the management of patients with digestive neuroendocrine tumours: well-differentiated tumour/carcinoma of the appendix and goblet cell carcinoma. Neuroendocrinology 2008; 87: 20-30.

[25] Gough DB, Donohue JH, Schutt AJ et al. Pseudomyxoma peritonei. Long-term patient survival with an aggressive regional approach. Ann Surg 1994; 219: 112-9.

[26] Paulman P, Klug R. Pseudomyxoma peritonei. Am Fam Physician 1986; 33: 173-4.

[27] Modlin IM, Lye KD, Kidd M. A 5-decade analysis of 13,715 carcinoid tumors. Cancer 2003; 97: 934-59.

[28] Kiran RP, Tripodi G, Frederick W, Dudrick SJ. Adenosquamous carcinoma of the colon: a rare tumor. Am Surg 2006; 72: 754-5.

[29] Dong Y, Wang J, Ma H, Zhou H, Lu G, Zhou X. Primary adenosquamous carcinoma of the colon: report of five cases. Surg Today 2009;39: 619-23.

[30] Frizelle FA, Hobday KS, Batts KP, Nelson H. Adenosquamous and squamous carcinoma of the colon and upper rectum: a clinical and histopathologic study. Dis Colon Rectum 2001; 44: 341-346. 


\section{Table legends}

\section{Table 1}

Demographic data and clinical presentation for all histological subtypes of appendiceal carcinoma

\section{Table 2}

Histopathological characteristics of appendiceal carcinoma according to histological subtype

Table 3

Pattern of metastatic spread for all histological subtypes of appendiceal carcinoma 


\section{Figure legends}

\section{Figure 1}

Overall 5-year survival according to histological subtype

\section{Figure 2}

5 -year DFS survival of carcinoid vs. noncarcinoid appendiceal tumors 
Table 1

Demographic data and clinical presentation for all histological subtypes of appendiceal carcinoma

\begin{tabular}{lcccc}
\hline & AC & aA & mAA & AAS \\
\hline Number (n/\%) & $48(24.5)$ & $99(50.5)$ & $45(23)$ & $4(2)$ \\
\hline Age (years) & $51.1 \pm 17.4$ & $64.7 \pm 12.0$ & $62.4 \pm 15.4$ & $56.2 \pm 8.5$ \\
\hline Male / female ratio & $1.17: 1$ & $1.67: 1$ & $0.96: 1$ & $3: 1$ \\
\hline BMI (kg/m) & & & & \\
\hline
\end{tabular}

Clinical presentation (\%)

- referral for complementary

$\begin{array}{lcccc}\text { surgery } & 22.9 & 34.3 & 24.4 & 25 \\ \text { acute appendicitis } & 37.5 & 36.4 & 37.8 & 50 \\ \text { incidential finding } & 29.1 & 22.2 & 20 & - \\ \text { nonspecific /tumor-related } & & & & \\ \text { symptoms } & 10.4 & 7.0 & 17.8 & 25\end{array}$

AC, appendiceal carcinoids; aA, appendiceal adenocarcinoma; mAA, mucinous appendiceal adenocarcinoma; AAS, appendiceal adenosquamous carcinoma

${ }^{1}$ Mean; standard deviation in parentheses 
Table 2

Histopathological characteristics of appendiceal carcinoma according to histological subtype

\begin{tabular}{lllcc}
\hline & AC & aA & mAA & AAS \\
\hline Overall cases (n) & 48 & 99 & 45 & 4 \\
Tumor size (cm) & & & & \\
T status & $1.8( \pm 1.64)$ & $3.27( \pm 2.01)$ & $4.0( \pm 3.05)$ & $3.1( \pm 1.41)$ \\
- T1 / T2 (n/\%) & - & & & \\
- T3 / T4 (n/\%) & - & $27(27.3)$ & $5(11.1)$ & - \\
N Status (pos. /\%) & $8(16.6)$ & $72(72.7)$ & $40(88.9)$ & $4(100)$ \\
Grading (n/\%) & & $36(36.4)$ & $12(26.7)$ & $2(50)$ \\
- G1 & - & & & \\
- G2 & - & $9(9.1)$ & $11(24.4)$ & - \\
- G3 & - & $61(61.6)$ & $21(46.7)$ & $1(25)$ \\
UICC stage (n/\%) & & $29(29.3)$ & $13(28.9)$ & $3(75)$ \\
- I & - & & & \\
- II & - & $15(15.1)$ & $3(6.7)$ & - \\
- III & - & $42(42.4)$ & $20(44.4)$ & $1(25)$ \\
- IV & - & $19(19.2)$ & $9(20)$ & $1(25)$ \\
\hline
\end{tabular}

AC, appendiceal carcinoids; aA, appendiceal adenocarcinoma; mAA, mucinous appendiceal adenocarcinoma; AAS, appendiceal adenosquamous carcinoma

${ }^{1}$ Mean; standard deviation in parentheses 
Table 3

Pattern of metastatic spread for all histological subtypes of appendiceal carcinoma

\begin{tabular}{lllll}
\hline $\begin{array}{l}\text { Anatomical site of } \\
\text { distant metastases }\end{array}$ & AC & aA & mAA & AAS \\
\hline Overall cases ( $\mathbf{n})$ & 48 & 99 & 45 & 4 \\
Metastases ( $\mathbf{n} / \%)$ & $2(4.2)$ & $23(23.2)$ & $13(28.9)$ & $2(50)$ \\
Site: & 1 & 10 & 1 & - \\
- Hepatic ( $\boldsymbol{n})$ & - & 2 & 3 & - \\
- Ovaries $(\boldsymbol{n})$ & 1 & 16 & 10 & 2 \\
- Peritoneal carcinomatosis $(\boldsymbol{n})$ & 16 & & \\
\hline
\end{tabular}

AC, appendiceal carcinoids; aA, appendiceal adenocarcinoma; mAA, mucinous appendiceal adenocarcinoma; AAS, appendiceal adenosquamous carcinoma 
Figure 1

Overall 5-year survival according to histological subtype

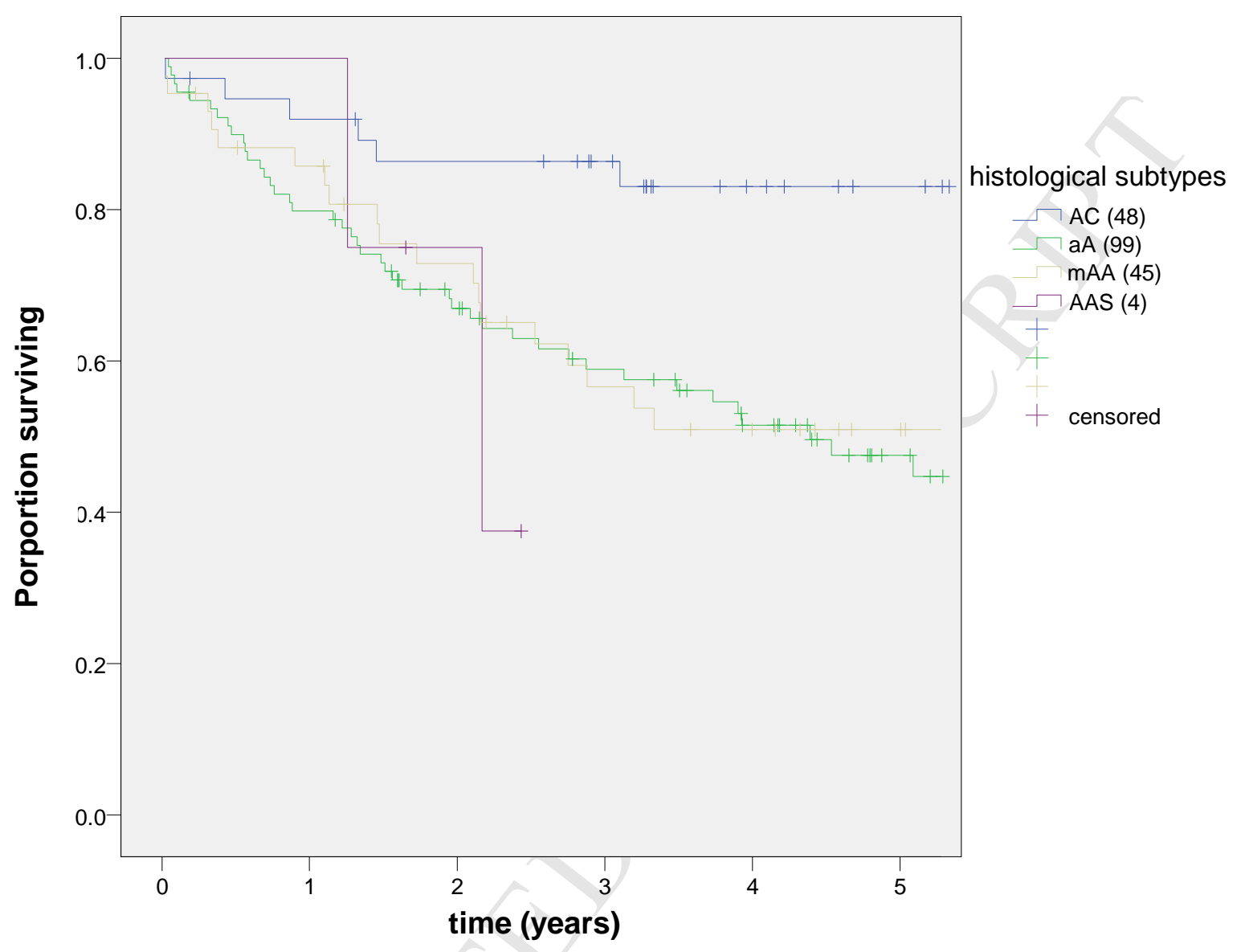

AC, appendiceal carcinoids; aA, appendiceal adenocarcinoma; mAA, mucinous appendiceal adenocarcinoma; AAS, appendiceal adenosquamous carcinoma 
Figure 2

5-year DFS survival of carcinoid vs. noncarcinoid appendiceal tumors

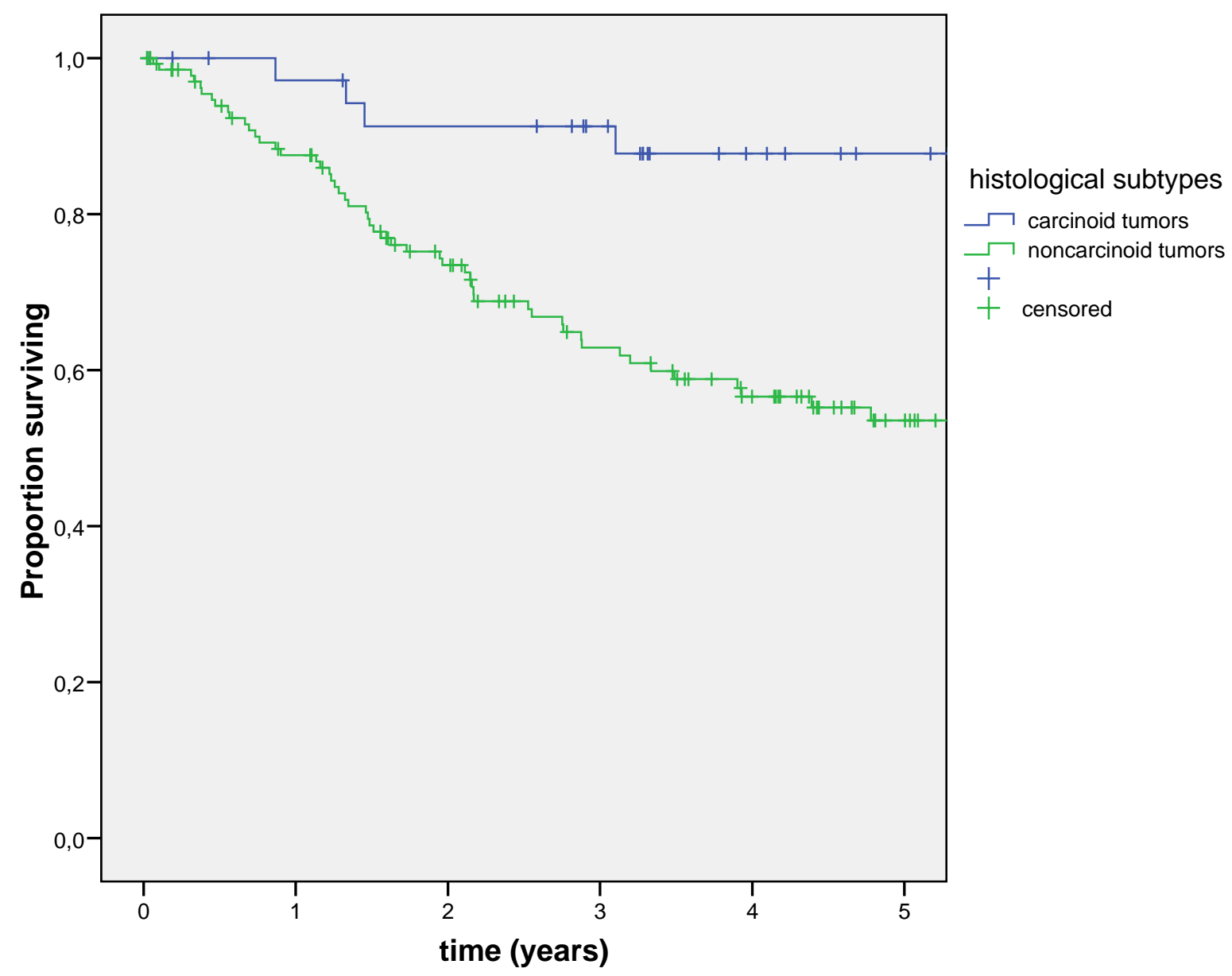

carcinoid tumors $(n=48)$; noncarcinoid tumors $(n=148)$ 